\title{
APPLICATIONS HOLOMORPHES DE DOMAINES DISQUÉS NON BORNÉS
}

\author{
JEAN-JACQUES LOEB
}

\begin{abstract}
We give several extensions to unbounded domains of the following classical theorem of $\mathrm{H}$. Cartan: A biholomorphism between two bounded complete circular domains of $\mathbb{C}^{n}$ which fixes the origin is a linear map. In our paper, pseudo-convexity plays a main role. Some precise study is done for the case of dimension two and the case where one of the domains is $\mathbb{C}^{n}$.
\end{abstract}

\section{Introduction}

Les domaines disqués de $\mathbb{C}^{n}$ ont d'abord été étudiés par H. Cartan vers 1930. Rappelons ici qu'un domaine dans $\mathbb{C}^{n}$ est dit disqué lorsque l'appartenance de $x$ à ce domaine implique l'appartenance de $z x$ à ce domaine pour tout complexe $z$ de module au plus un. Pour $n=1$, les seuls domaines disqués sont les disques ou $\mathbb{C}$ tout entier. La situation est beaucoup plus riche en dimension supèrieure.

Un des plus anciens résultats dû à H. Cartan [4] est le suivant. (Son énoncé original est plus fort que l'énoncé que nous donnons.)

Résultat 1 (R1). Soit $f$ un biholomorphisme d'un domaine disqué $X_{1}$ sur un autre domaine disqué $X_{2}$ envoyant l'origine sur l'origine. Si un des deux domaines est supposé borné, alors ce biholomorphisme est un automorphisme linéaire.

Ce théorème est conséquence du résultat suivant (dû également à H. Cartan).

Résultat 2 (R2). Soit $V$ un domaine borné de $\mathbb{C}^{n}$ et $f$ une application holomorphe de $V$ dans lui-même. On suppose que $f$ a un point fixe a et qu'en ce point fixe, la différentielle de $f$ soit l'idendité. Alors $f$ elle-même est l'idendité.

2000 Mathematics Subject Classification. Primary: 32A07; Secondary: 32H02, $32 \mathrm{~T} 05$.

Key words. Complete circular domains, self holomorphic map. 
Un troisième résultat dû à $\mathrm{W}$. Rudin $[\mathbf{1 3}]$ est le suivant (voir aussi pour le cas de la boule M. Hervé [7]).

Résultat 3 (R3). Soit $U$ un domaine borné de $\mathbb{C}^{n}$ supposé strictement convexe, disqué et borné et $f$ une auto-application holomorphe de $U$ laissant fixe l'origine. Alors l'ensemble des points fixes de $f$ est l'intersection du sous-espace vectoriel de $\mathbb{C}^{n}$ des points fixés par $D f(0)$ (différentielle de $f$ en 0 ) avec $U$.

Dans la suite, nous nous proposons de donner certaines généralisations de ces théorèmes au cas disqué non borné. Au départ, faisons quelques remarques simples :

Lien entre les Résultats 1, 2 et 3. Rappelons que H. Cartan a montré que le Résultat 2 implique le Résultat 1 de la manière suivante : Supposons dans le Résultat 1 le domaine $X_{1}$ borné. Notons $g$ l'application réciproque de $f$. Alors pour $u$ complexe de module 1, l'application $h_{u}: x \rightarrow g\left(u^{-1} f(u x)\right)$ est une auto-application holomorphe de $X_{1}$ dont on vérifie facilement les hypothèses du Résultat 2 , à savoir : $h_{u}$ fixe l'origine et sa différentielle en ce point est l'idendité. On déduit que $h_{u}$ est l'idendité pour tout $u$ de module 1 , ce qui signifie aussi que $f(u x)=u f(x)$ pour tout $x$ dans $X_{1}$ et tout $u$ de module 1 . On montre alors facilement que $f$ est linéaire.

Il est aussi clair que le Résultat 3 implique le Résultat 2 dans le cas très particulier où $V$ satisfait aux conditions du Résultat 3 et lorsque $a$ est l'origine.

\section{Discussion des hypothèses.}

1. Le contre-exemple classique au Résultat 1 et au Résultat 2 dans le cas non borné est le suivant: On prend $X_{1}=X_{2}=\mathbb{C}^{2}$ et $f$ une application de la forme $:(x, y) \rightarrow(x, y+l(x))$ avec $l$ une fonction entière non nulle vérifiant $l(0)=l^{\prime}(0)=0$. Nous allons montrer dans la suite que malgré ce contre-exemple, on peut récupérer une certaine partie de le Résultat 1 dans le cas non borné.

2. Dans le cas du théorème de Rudin, on a des exemples de domaines bornés disqués pour lesquels le Résultat 3 est mis en défaut. On prend par exemple pour domaine le bidisque et pour $f$ l'application qui à $(x, y)$ associe $\left(x, x^{2}\right)$. Nous essayons ici de mieux comprendre cette situation.

3. La preuve donnée par H. Cartan de le Résultat 2 est de supposer $f$ différent de l'idendité. Son développement en polynômes homogènes à l'origine est alors de la forme : $f(x)=x+P(x)+\cdots$ 
avec $P$ le polynôme homogène non nul de degré le plus bas et supérieur à un. Le développement analogue du $n$-ième itéré est donné par : $x+n P(x)+\cdots$, ce qui viole les inégalités de Cauchy si on suppose $V$ borné.

Le théorème de Cartan a été généralisé au cas des variétés hyperboliques complexes [1]. En ce qui concerne les domaines disqués, ceci n'apporte rien de plus car un domaine disqué hyperbolique est borné [11].

Des exemples simples de domaines disqués non bornés sont des ensembles du type : $\left\{z \in \mathbb{C}^{n}|| P(z) \mid<1\right\}$ où $P$ est un polynôme homogène. Afin de mettre en évidence certains phénomènes, nous donnons ici deux classes d' exemples plus compliqués de domaines disqués pseudoconvexes et non bornés.

Exemple 1. On part d'une suite d'éléments $\left(a_{n}, b_{n}\right) \in \mathbb{C}^{2}$ vérifiant : $a_{n}$ non nul pour tout $n,\left|a_{n}\right|+\left|b_{n}\right|=1$ pour tout $n$ et l'ensemble des directions pointées par les vecteurs $\left(a_{n}, b_{n}\right)$ est dense dans l'ensemble des directions complexes.

On choisit alors, ce qui est possible, une suite de nombres $\left(c_{n}\right)$ strictement positifs tel que la somme des $c_{n} \ln \left|a_{n}\right|$ converge (i.e. soit strictement supérieur à $-\infty$ ). On peut aussi s'arranger pour que la somme de la série de terme général $c_{n}$ soit égal à un. Nous affirmons alors que la série de terme général $c_{n} \ln \left|a_{n} x+b_{n} y\right|$ converge ou vaut $-\infty$ pour tout $(x, y) \in \mathbb{C}^{2}$ et définit une fonction plurisousharmonique (psh) $h$ qui vérifie $: h(a v)=\ln |a|+h(v)$ pour tout complexe $a$ et tout $v \in \mathbb{C}^{2}$. On remarque d'abord que lorsque $\max (|x|,|y|)<1$, le terme général de la série précédente est négatif, donc il y a convergence (éventuellement vers $-\infty)$. Les fonctions $\ln \left|a_{n} x+b_{n} y\right|$ étant aussi psh, il s'en suit que $h$ est psh comme limite d'une suite décroissante de fonctions psh au moins lorsque $\max (|x|,|y|)<1$. Le passage à tout $\mathbb{C}^{2}$ se fait en utilisant la relation $\ln |x y|=\ln |x|+\ln |y|$, et permet aussi de montrer la relation vérifiée par $h$. La fonction $h$ est différente de $-\infty$ en $(1,0)$. Le domaine disqué $X$ considéré est alors défini comme étant l'ensemble des éléments $v$ de $\mathbb{C}^{2}$ vérifiant $h(v)<0$. C'est un domaine pseudo-convexe qui par construction contient un ensemble dense de droites vectorielles. On pourra remarquer que ceci implique l'inexistence de fonctions psh continues bornées non constantes sur $X$ (et à fortiori de fonctions holomorphes bornées non constantes) mais qu'il existe une fonction psh bornée non constante sur $X$ à savoir $h$.

Exemple 2. C'est un type de domaine construit en prenant l'intersection d'un domaine $X$ de l'Exemple 1 avec le domaine $Z=\left\{(x, y) \in \mathbb{C}^{2}\right.$ $|y|<1\}$. Il est disqué, pseudo-convexe, non borné et ne contient pas de 
droite vectorielle car l'axe des $x$ (qui est la seule droite vectorielle incluse dans $Z$ ) n'est pas dans $X$ par construction.

Des résultats de Th. J. Barth [2] concernant l'indicatrice de Kobayashi jouent dans nôtre article un rôle important (Parties 1 et 2). L'idée de disque dans le bord (Partie 3) avait été développée par E. Vesentini [14] pour les boules. Nos méthodes concernant la fonction de Green (Partie 4) sont proches de celles de F. Berteloot et G. Patrizio [3].

\section{2. Équivalence holomorphe des domaines disqués pseudo-convexes}

Le théorème suivant est un corollaire immédiat de le Résultat 1 dans le cas borné.

Théorème 1. Soit $f$ un biholomorphisme entre deux domaines disqués dont l'un est pseudo-convexe et qui conserve l'origine. Alors les deux domaines sont linéairement équivalents par $D f(0)$.

Nous ignorons si ce théorème demeure vrai lorsqu'on supprime l'hypothèse de pseudo-convexité. Notons simplement que l'enveloppe d'holomorphie d'un domaine disqué est univalente et disquée [12], ce qui permet de prolonger le biholomorphisme de départ en un biholomorphisme des enveloppes mais ne permet pas de conclure.

\subsection{Lien avec l'indicatrice de Kobayashi.}

Aprés avoir donné une preuve directe du résultat précédent, nous avons constaté qu'on pouvait le déduire aisément du résultat de Th. J. Barth [2] :

Résultat 4. L'indicatrice de Kobayashi d'un domaine disqué pseudoconvexe à l'origine est le domaine lui-même.

Remarque. D'aprés ce théorème et en utilisant l'Exemple 2, on voit qu'il existe des indicatrices de Kobayashi non bornées et sans droite vectorielle.

En vue de ce qui suit, rappelons briévement certaines définitions et résultats [9] :

Définition 1. Soit $U$ un domaine étoilé de $\mathbb{R}^{n}$ (c'est à dire un domaine tel que si $x \in U$, alors $t x \in U$ pour tout $t \in[0,1])$. La jauge $j_{U}$ associée à $U$ est une fonction positive sur $\mathbb{R}^{n}$ définie par :

$$
j_{U}(x)=\inf \{t>0 \mid x / t \in U\} .
$$


Cette fonction est semi-continue supérieurement (scs) et on a : $U=\{x \mid$ $\left.j_{U}(x)<1\right\}$.

Si $U$ est disqué dans $\mathbb{C}^{n}$, alors $j_{U}(z x)=|z| j_{U}(x)$ pour tout complexe $z$ et tout $x \in \mathbb{C}^{n}$.

La propriété suivante est également démontrée dans [2].

Résultat 5. Un domaine disqué de $\mathbb{C}^{n}$ est pseudo-convexe si et seulement si sa jauge est logarithmiquement plurisousharmonique (log-psh).

Définition 2. Soit $M$ une variété complexe et $a \in M$. On note $E_{a}$ l'ensemble des applications holomorhes $f$ définies sur le disque unité et à valeurs dans $M$ telles que $f(0)=a$. Alors par définition l'indicatrice de Kobayashi en $a$, notée $K_{a}(M)$, est le sous-ensemble de l'espace tangent en $a$ dont les éléments sont de la forme $r f^{\prime}(0)$ avec $r \in\left[0,1\right.$ [ et $f \in E_{a}$.

On montre facilement [9] que l'indicatrice de Kobayashi en un point est un domaine disqué dans l'espace tangent.

On a :

Proposition 1. Soit $f$ une application holomorphe d'un domaine disqué $X$ à valeurs dans un domaine disqué $Y$ pseudo-convexe, et qui envoie l'origine en l'origine. Alors $D f(0)(X) \subseteq Y$.

Preuve: Soit $v \in X$. Il existe $p>1$ tel que la fonction $h: z \rightarrow f(z p v)$ soit définie et holomorphe sur le disque unité $\Delta$ et à valeurs dans $Y$. On déduit que $(1 / p) h^{\prime}(0)=D f(0) v$ est dans $K_{0}(Y)$ par définition de l'indicatrice de Kobayashi. Le théorème de Barth dit que $K_{0}(Y)=Y$, d'où le résultat.

Preuve du Théorème 1: On sait qu'un biholomorphisme transforme domaine pseudo-convexe en domaine pseudo-convexe $[6]$. On applique alors la proposition précédente au biholomorphisme et son inverse pour conclure.

\subsection{Le cas de $\mathbb{C}^{n}$.}

Un corollaire immédiat du théorème précédent est qu'un domaine disqué $Y$ biholomorphe à $\mathbb{C}^{n}$ est $\mathbb{C}^{n}$ lui-même. En effet, quitte à composer par une translation de $\mathbb{C}^{n}$, on peut supposer l'origine fixée. La pseudoconvexité de $\mathbb{C}^{n}$ permet de conclure. Ce résultat qui se traduit par la non existence d'un domaine de Fatou-Bieberbach disqué peut être renforcé par le théorème suivant : 
Théorème 2. Soit $X$ un domaine disqué dans $\mathbb{C}^{n}$. Alors $X$ est nécessairement égal à $\mathbb{C}^{n}$ dans les cas suivants :

1) Il existe une application holomorphe propre de $X$ dans $\mathbb{C}^{n}$.

2) Il existe une application holomorphe propre de $\mathbb{C}^{n}$ dans $X$.

3) $X$ est pseudo-convexe et il existe une application holomorphe non dégénérée (i.e. de Jacobien non identiquement nul) de $\mathbb{C}^{n}$ dans $X$.

Avant de passer à la preuve, nous faisons les remarques suivantes :

a) Pour 1), il nous parait difficile d'affaiblir la condition de propreté, même dans le cas $n=1$ et $Y=\Delta$.

b) Le 3) peut être pris en défaut si $X$ n'est pas pseudo-convexe. On prend pour $X$ le complémentaire dans $\mathbb{C}^{2}$ de l'ensemble des $(0, b)$ avec $|b| \geq 1$. L'application $(x, y) \rightarrow(x, x y)$ est une application holomorphe non dégénérée de $\mathbb{C}^{2}$ dans $X$.

Preuve: Le point essentiel ici est qu'il existe sur un domaine disqué qui n'est pas l'espace entier, une fonction log-psh (et donc psh) bornée et non constante à savoir la jauge. Or une telle fonction n'existe pas pour $\mathbb{C}^{n}$ (théorème de Liouville $[\mathbf{8}]$ ).

Preuve de 1): On sait que si on a une application holomorphe propre entre deux domaines de $\mathbb{C}^{n}$, la pseudo-convexité d'un des domaines implique la pseudo-convexité de l'autre [6]. Donc $X$ est pseudo-convexe. Supposons $X$ différent de $\mathbb{C}^{n}$ et notons $j$ la jauge de $X$. Soit $f$ une application holomorphe propre de $X$ dans $\mathbb{C}^{n}$. Pour $t$ dans $\mathbb{C}^{n}$, on pose : $h(t)=\max _{s \in F_{t}} j(s)$ où on note $F_{t}$ la fibre de l'application $f$ au-dessus de $t$. D'après un théorème de Klimek [8, p. 73], la fonction $h$ est psh sur $\mathbb{C}^{n}$. Elle est évidemment bornée. De plus, elle est non constante car $j$ sans atteindre la valeur 1 sur $X$ prend des valeurs aussi proche de 1 qu'on veut. On a donc une contradiction avec le théorème de Liouville et donc $X=\mathbb{C}^{n}$.

3) implique 2) car on a vu que $X$ est nécessairement pseudo-convexe. De plus une application holomorphe propre est non dégénérée.

Preuve de 3): Soit $f$ une application non dégénérée de $\mathbb{C}^{n}$ dans $X$ supposé distinct de $\mathbb{C}^{n}$. La fonction $j \circ f$ (avec $j$ jauge de $X$ ) est psh sur $\mathbb{C}^{n}$ et donc égale à une constante $c$ comprise entre 0 et 1 . Comme $f$ est non dégénérée, ceci implique que $j$ vaut $c$ sur un ouvert non vide de $X$.

Premier cas : $c=0$. La fonction $j$ étant log-psh et non identiquement nulle, on déduit que l'ensemble $\{j=0\}$ est pluripolaire et donc d'intérieur vide. Ce cas ne peut se produire. 
Deuxième cas : $c \neq 0$. Alors les ensembles $\{j=s\}$ avec $s \neq 0$ forment une famille non dénombrable d'ensembles disjoints deux à deux et d'intérieurs non vides comme homothétiques de $\{j=c\}$, ce qui est impossible.

\subsection{Remarques sur le cas quasi-disqué.}

Les résultats du théorème précédent restent vrais si on remplace disqué par quasi-disqué. Faisons quelques rappels : On se donne $d=\left(d_{1} \cdots d_{n}\right)$ un $n$-uplé de nombres entiers strictement positifs et premiers entre eux. Pour $x=\left(x_{1}, \ldots x_{n}\right) \in \mathbb{C}^{n}$ et $t \in \mathbb{C}$, on pose : $t^{d} x=\left(t^{d_{1}} x_{1}, \ldots, t^{d_{n}} x_{n}\right)$. Un domaine $X$ dans $\mathbb{C}^{n}$ est appelé quasi-disqué de type $d$ si pour tout $x \in X$ et tout $t$ complexe de module au plus un, on a : $t^{d} x \in X$. La jauge $j_{X}$ pour un quasi-disqué $X$ de type $d$ se définit de la même manière que pour un disqué, à savoir que pour $v \in \mathbb{C}^{n}$, on a : $j_{X}(v)=\inf \{t>$ $\left.0 \mid\left(t^{-1}\right)^{d} v \in X\right\}$. On vérifie que $X=\left\{v \mid j_{X}(v)<1\right\}$. La jauge est semi-continue supérieurement et on a également pour tout complexe $t$ et tout $v \in \mathbb{C}^{n}: j_{X}\left(t^{d} v\right)=|t| j_{X}(v)$. La proposition suivante généralise le théorème de Barth :

Proposition 2. La jauge $j_{X}$ est log-psh si et seulement si $X$ est pseudoconvexe.

Nous ne donnerons pas la preuve qui est une simple adaptation de celle donnée par Barth dans le cas disqué. La généralisation du Théorème 2 au cas quasi-disqué s'en déduit.

\section{Lemme de Schwarz et applications}

Nous donnons ici un lemme de Schwarz dû à A. Sadullaev [10]. Pour être complet, nous en donnons ici la preuve. Ce lemme permet d'établir certaines généralisations de le Résultat 2 et le Résultat 3 au cas disqué non borné.

Dans le cas de la dimension deux, nous arrivons à un résultat presque optimal concernant le Résultat 2.

Lemme 1. Soit $f$ une application holomorphe de $\Delta$ à valeurs dans un domaine disqué $X$ pseudoconvexe et vérifiant $f(0)=0$. Alors on a:

1) $j_{X} \circ f(z) \leq|z|$.

2) Si l'inégalité devient une égalité en un point $z \neq 0$ ou si $j\left(f^{\prime}(0)\right)=$ 1 , alors l'inégalité précédente devient une égalité pour tout z dans $\Delta$ et nécessairement on $a: j_{X}\left(f^{\prime}(0)\right)=1$.

Preuve: L'application $f(z) / z$ est holomorphe sur $\Delta$. On voit que $j_{X}(f(z) / z)=\frac{j_{X}(f(z))}{|z|}$ est plus petit que $1 / r$ pour $|z|=r(r<1)$. 
Le principe du maximum appliqué à la fonction psh $j_{X}(f(z) / z)$ montre que cette fonction est plus petite que $1 / r$ pour $|z| \leq r$. En faisant tendre $r$ vers 1 , on obtient le 1). Les hypothèses du 2) signifient que la fonction psh $j_{X}(f(z) / z)$ atteint son maximum 1 en un point de $\Delta$ donc elle est constante ce qui termine la preuve.

Discussion du cas de l'égalité. On suppose qu'on a l'égalité dans le lemme précédent. Deux situations se présentent : a) $f(z)=z f^{\prime}(0)$ pour tout $z \in \Delta$. b) si a) n'est pas vérifié, alors nécessairement, il existe une application holomorphe non constante $h$ de $\Delta$ à valeurs dans $X$ telle que $j \circ h$ soit identiquement 1 .

En vue de ce qui suit,on introduit l'hypothèse $(\mathrm{H})$ suivante :

(H) Un disqué $X$ satisfait à $(\mathrm{H})$ si pour toute application holomorphe : $f: \mathbb{C} \rightarrow X$ (qu'on appellera application entière par la suite) et vérifiant $f(0)=0$, l'image de $f$ (qu'on appellera courbe entière par la suite) est contenue dans une droite vectorielle.

Le cas de la dimension deux fait l'objet de la proposition suivante :

Proposition 3. Un disqué pseudoconvexe $X$ de $\mathbb{C}^{2}$ et différent de $\mathbb{C}^{2}$ satisfait $(H)$

Preuve: Si $f$ est une application entière à valeur dans $X$, alors par Liouville, $j_{X} \circ f$ est constante. Si de plus $f(0)=0$, alors $j_{X} \circ f$ est identiquement nulle. Par l'homogénéité de $j_{X}$, le sous-ensemble $S$ de $\mathbb{C}^{2}$ défini par : $S:=\{a f(z) \mid a \in \mathbb{C}, z \in \mathbb{C}\}$ est contenu dans $\left\{j_{X}=0\right\}$ et cet ensemble est pluripolaire car on a : $X \neq \mathbb{C}^{2}$ et $j_{X} \log$-psh. Supposons maintenant que $f(\mathbb{C})$ ne soit pas contenu dans une droite complexe. En notant $p$ la projection canonique de $\mathbb{C}^{2}-(0)$ sur la droite projective, on voit que $p(f(\mathbb{C}))$ est d'intérieur non vide. Il en est alors de même de $S$, ce qui est contradictoire avec sa pluripolarité. Donc $(\mathrm{H})$ est bien vérifiée pour $X$

Par la suite, on notera de manière générale $p$ la projection canonique de $\mathbb{C}^{n}-(0)$ sur l'espace projectif $\mathbb{P}^{n-1}$.

Avant d'introduire la définition principale de ce paragraphe, on fait la remarque élémentaire suivante : Soit $X$ un domaine disqué de dimension $n$. On se donne une direction $d$ de $\mathbb{C}^{n}$ qu'on peut voir comme élément de $\mathbb{P}^{n-1}$, et qu'on peut écrire sous la forme $d=p(v)$. Si $j_{X}(v)$ est non nul, on peut quitte à changer $v$ supposer que $j_{X}(v)=1$. Dans le cas opposé on aura $j_{X}(v)=0$ pour tout représentant. On dira dans ce dernier cas que $d$ est une direction entière. 
Définition 3. Soit $X$ un domaine disqué pseudo-convexe et vérifiant $(\mathrm{H})$. On dira qu'une direction $d$ est bonne dans les deux cas suivants :

1) $d$ est une direction entière.

2) $d=p(v)$ avec $j_{X}(v)=1$ et il n'existe pas d'application holomorphe non constante $f$ de $\Delta$ dansl'ensemble $\left\{x \mid j_{X}(x)=1\right\}$ avec $f(0)=$ $v$. (On peut exprimer ceci en disant qu'il n'existe pas de vrai disque analytique contenu dans l'ensemble $\left\{j_{X}=1\right\}$ et passant par $v$.)

Exemple 3. Si $X$ est le bidisque $\Delta \times \Delta$, alors les bonnes directions sont de la forme $p((u, v))$ avec $u$ et $v$ de module 1 .

On donne maintenant les généralisations des Résultats 2 et 3 .

Dans la proposition et le théorème qui suit, on notera $X$ un domaine disqué pseudo-convexe dans $\mathbb{C}^{n}$.

Proposition 4. Soit $f$ une auto-application holomorphe de $X$ fixant l'origine. On a pour tout $x \in X: j_{X}(f(x)) \leq j_{X}(x)$.

De plus si $D f(0) x=x$ pour un certain $x$, on $a: j_{X}(f(x))=j_{X}(x)$.

En particulier, si $D f(0)$ vaut l'idendité, alors l'inégalité précédente devient une égalité pour tout $x$ dans $X$.

\section{Théorème 3 .}

A) On suppose l'ensemble des bonnes directions de $X$ non inclus dans un sous-ensemble algébrique propre de $\mathbb{P}^{n-1}$ et que $X$ vérifie $(H)$, alors :

(R2) Toute auto-application holomorphe de $X$ conservant l'origine et tangent à l'idendité en ce point vaut l'idendité.

(R1) Tout biholomorphisme d'un domaine disqué sur $X$ et conservant l'origine est linéaire.

B) On suppose que $X$ ne contient pas de droite complexe vectorielle et que toutes les directions sont bonnes, alors :

(R3) L'ensemble des points fixes d'une auto-application holomorphe conservant l'origine est l'intersection de $X$ et du sous-espace vectoriel des points fixés par $D f(0)$.

Remarque. Si $n=2$, la condition A) signifie simplement qu'il y a un nombre infini de bonnes directions. 
Preuve de la Proposition 4: Supposons d'abord que $j_{X}(x)=0$. Alors on a une application entière $z \rightarrow f(z x)$ à valeurs dans $X$ et ceci implique comme on l'a déjà vu que $j_{X}(f(z x))$ est identiquement nul. En particulier $j_{X}(f(x))$ est nul. La proposition est alors évidente dans ce cas.

Pour l'autre cas, on applique le lemme de Schwarz à la fonction $z \rightarrow$ $f(z v)$ en supposant $j_{X}(v)=1$.

En vue de la preuve du théorème, on utilise le lemme :

Lemme 2. Soit $f$ une application holomorphe d'un domaine disqué de $\mathbb{C}^{n}$ à valeurs dans $\mathbb{C}^{n}$. Alors l'ensemble des droites complexes vectorielles qui sont envoyées sur elle-mêmes forment (par projection canonique) un sous-ensemble algébrique de $\mathbb{P}^{n-1}$.

Preuve: On écrit que deux vecteurs de $\mathbb{C}^{n}$ sont linéairement dépendants en écrivant que tous les deux-mineurs de la matrice formée par les deux vecteurs sont nuls. On écrit ensuite que la droite vectorielle définie par $x \neq 0$ est envoyée sur elle-même si et seulement si $x$ et $f(a x)$ sont linéairement dépendants pour tout $a \in \Delta$. Le développement à l'origine de $f$ en série d'applications polynomiales homogènes montre que la condition précédentes se traduit par la nullité en $x$ d'une famille de polynômes homogènes, ce qui est la conclusion désirée.

Preuve du Théorème 3: Prouvons d'abord A) (R2). Soit $d$ une bonne direction.

Si $d$ n'est pas entière, on écrit $d=p(v)$ avec $j_{X}(v)=1$. D'après la proposition précédente, on a $j_{X}(f(z v))=|z|$ pour tout $z \in \Delta$ et comme $d$ est une bonne direction, ceci implique que $f(z v)=z v$ pour tout $z \in \Delta$.

Soit ensuite le cas $d=p(x)$ entière. On considère l'application entière $z \rightarrow f(z x)$ à valeurs dans $X$. L'hypothèse $(\mathrm{H})$ jointe à $D f(0)=$ $i d$ permet de conclure que $f(z x)$ est à valeur dans la droite vectorielle engendrée par $x$.

Le lemme précédent joint à l'hypothèse sur les bonnes directions montre que toutes les droites vectorielles sont envoyées dans elle-mêmes. Il existe alors une fonction $a$ définie sur $X$ dont on vérifie facilement qu'elle est holomorphe et telle que : $f(x)=a(x) x$ pour tout $x \in X$. L'hypothèse $D f(0)=i d$ implique $a(0)=1$ et d'après la proposition précédente, on a aussi : $J_{X}(f(x))=J_{X}(x)$ pour tout $x$. On en déduit d'abord que le module de $a$ est constant égal à un puis que $a$ elle-même est identiquement égale à un, ce qui termine la preuve de (R2). La preuve de (R1) est alors exactement celle donnée par H. Cartan.

B) (R3). Pour $v$ telle que $j_{X}(v)=1$, on applique le lemme de Schwarz à la fonction $z \rightarrow f(z v)$ et on conclut grâce à l'hypothèse. 
On peut renforcer $(\mathrm{R} 2)$ par la proposition suivante :

Proposition 5. Soit $X$ un domaine pseudo-convexe disqué vérifiant (R2), et $f$ une auto-application holomorphe propre fixant l'origine et telle que $D f(0)$ soit diagonalisable avec ses valeurs propres de modules tous égaux à 1 . Alors $f$ est linéaire.

Pour la preuve, on utilise le lemme élémentaire suivant :

Lemme 3. Soit $A$ un automorphisme linéaire de $\mathbb{R}^{n}$ qui envoie un ouvert $X$ dans lui-même. On suppose $A$ diagonalisable (au sens complexe) avec ses valeurs propres de module 1 . Alors $A(X)=X$.

Preuve du Lemme 3: Remarquons d'abord que le sous-ensemble formé des matrices de $G l(n, \mathbb{R})$ qui envoient $X$ dans lui-même est fermé dans $G l(n, \mathbb{R})$. En effet $B$ est dans ce sous-ensemble si et seulement si $B^{-1}$ envoie le complémentaire de $X$ (qui est fermé) dans lui-même.

Soit maintenant $A$ vérifiant les hypothèses du lemme. Par diagonalisation, il est facile de voir qu'il existe une suite de puissances positives de $A$ qui tend vers la matrice idendité, et donc il existe une suite de puissances de $A$ qui tend vers $A^{-1}$. D'après ce qui a été dit au début, on a donc $A^{-1}(X)$ inclus dans $X$ et donc aussi $A(X)=X$.

Preuve de la Proposition 5: D'après la Proposition 1, l'application linéaire $A=D f(0)$ envoie $X$ dans $X$, donc d'après le lemme précédent, ceci reste vrai pour $A^{-1}$. On applique (R2) à $A^{-1} \circ f$ pour conclure.

Remarque. Pour un domaine borné ou plus généralement une variété complexe hyperbolique, la différentielle d'une auto-application holomorphe en un point fixe a ses valeurs propres de module au plus un. Ceci peut être mis en défaut pour un domaine disqué $D$ pseudo-convexe vérifiant (R2). On prend $D=\left\{(x, y) \in \mathbb{C}^{2}|| x y|<1| x \mid,<1\right\}$ et pour $f$ l'application linéaire qui à $(x, y)$ associe $(x / 2,2 y)$. Le fait que $D$ vérifie (R2) est une conséquence du théorème du chapitre suivant.

Donnons pour finir ce chapitre, des exemples de domaines pseudoconvexes disqués et non bornés qui vérifient les hypothèses données pour (R3). On note $j$ la jauge d'un domaine de l'Exemple 1 et $s$ la jauge d'un domaine de type défini dans l'Exemple 2. Soit $X$ un domaine disqué défini par la jauge $j+s$. Il est pseudoconvexe car $j$ et $s$ sont psh et ne contient pas de droite vectorielle car $s(x)>0$ en dehors de l'origine (un domaine de type défini dans l'Exemple 2 ne contenant pas de droite vectorielle). De plus, il est non borné. En effet ceci signifie que la restriction de $j+s$ à la sphère $S^{3}$ peut être rendue arbitrairement petite 
en un point. Cette assertion se prouve en utilisant que pour $\epsilon>0$ l'ensemble $\left\{x \in S^{3} \mid s(x)<\epsilon\right\}$ est un ouvert non vide et que $j$ s'annule sur un ensemble dense de droites vectorielles. Reste à montrer que toutes les directions sont bonnes. Dans le cas contraire $\ln (j+s)$ serait une fonction pluriharmonique sur un ouvert non vide $W$ (voir paragraphe suivant). On écrit alors en se restrignant à $W: j=\exp (u)$ et $s=\exp (v)$ et $j+s=\exp (w)$ avec $u$ et $v$ psh et $w$ pluriharmonique. Ceci implique en particulier que $u$ est pluriharmonique sur $W$. Mais c' est impossible car par construction de l'Exemple 1, $u$ vaut $-\infty$ sur un ensemble dense.

\section{Le cas de la dimension deux}

Comme nous l'avons annoncé, nous donnons un résultat précis en dimension deux. Pour ceci, nous introduisons :

Définition 4. Les domaines disqués pseudo-convexes exceptionnels sont $\mathbb{C}^{2}$ et les domaines définis de la manière suivante :

On se donne $n$ forme linéaires $f_{1}, \ldots, f_{n}$ non nulles sur $\mathbb{C}^{2}$ (on supposera aussi par convenance pour la suite qu'elles sont non proportionnelles deux à deux), et $n$ nombres réels strictement positifs : $a_{1}, \ldots, a_{n}$. Le domaine associé est alors défini par :

$$
\left\{\left.(x, y) \in \mathbb{C}^{2}\left|\prod_{i=1}^{n}\right| f_{i}\right|^{a_{i}}<1\right\} .
$$

On dira aussi que le domaine exceptionnel est du premier type s'il est égal à $\mathbb{C}^{2}$ ou s'il est défini à partir d'une ou deux formes linéaires et du second type sinon.

Voici le théorème principal :

Théorème 4. Tous les domaines disqués pseudo-convexes satisfont au théorème d'unicité de Cartan à l'origine (propriété (R2)) à l'exception éventuelle des domaines exceptionnels.

Remarque. Il est aisé de montrer que les domaines du premier type ne satisfont pas au théorème d'unicité. Le cas de $\mathbb{C}^{2}$ a été vu. Le cas $n=1$ (produit du disque par $\mathbb{C}$ ) se traite de la même manière. Pour $n=2$, on se rammène au cas $f_{1}=x$ et $f_{2}=y$ et on considére des applications : $(x, y) \rightarrow\left(\exp \left(-a_{2} h(x, y)\right) x, \exp \left(a_{1} h(x, y)\right) y\right.$ avec $h$ une fonction entière sur $\mathbb{C}^{2}$ nulle à l'origine.

Preuve du Théorème 4: Pour la preuve, nous utilisons certains résultats de la théorie du pluripotentiel (voir [5]). On considère un domaine disqué pseudo-convexe $X$ dans $\mathbb{C}^{2}$. On note $G_{X}$ la fonction de Green du domaine 
qui est par définition le logarithme de la jauge. On sait que $d d^{\mathbb{C}} G_{X}$ est le pull-back d'une mesure de probabilité $m$ sur $\mathbb{P}^{1}$ et que la donnée de $m$ caractérise de manière unique à une constante additive près la fonction de Green $G_{X}$. On fait alors la remarque suivante : Si $d \in \mathbb{P}^{1}$ n'est pas une bonne direction, il s'en suit que la fonction de Green $G_{X}$ est pluriharmonique au voisinage d'un $x_{0}$ de $\mathbb{C}^{2}$ qui pointe dans la direction $d$. En utilisant une section locale $s$ de $\mathbb{P}^{1}$ dans $\mathbb{C}^{2}-(0)$ qui envoie $d$ sur $x_{0}$, ceci signifie aussi que $G_{X} \circ s$ est localement pluriharmonique, ou que le $d d^{\mathbb{C}}$ de cette fonction est nul et donc la mesure $m$ associée ne charge pas la direction $d$.

On a vu que si un domaine disqué pseudo-convexe ne satisfait pas au théorème d'unicité de Cartan, alors le nombre de ses bonnes directions est fini. Mais ceci implique alors que $m$ est une combinaison linéaire finie à coefficients positifs de masse de Dirac sur $\mathbb{P}^{1}$. En écrivant explicitement $m$ sous la forme $\sum_{i} a_{i} \delta_{d_{i}}$ et en posant $d_{i}=p\left(\left(b_{i}, c_{i}\right)\right)$, on voit qu'une solution $G$ satisfaisant à l'équation $d d^{\mathbb{C}} G=m$ est de la forme $\sum_{i} a_{i} \ln \left|b_{i} x+c_{i} y\right|$. L'unicité permet de conclure.

Pour les domaines exceptionnels du second type, nous avons le résultat partiel suivant :

Proposition 6. Un domaine exceptionnel (voir Définition 4) défini à partir de $n$ formes linéaires $f_{i}($ avec $n \geq 3)$ et par des $a_{i}$ tous égaux à 1 vérifie (R2).

Preuve: Faisons d'abord la remarque générale suivante : Soient $P$ un polynôme homogène sur $\mathbb{C}^{n}$ et $X$ le domaine défini par : $\left\{x \in \mathbb{C}^{n} \mid\right.$ $|P(x)|<1\}$. Soit $g$ une auto-application holomorphe du domaine $X$ laissant fixe l'origine et tangente à l'idendité en ce point. Le domaine $U$ est pseudo-convexe disqué et sa jauge est une puissance de $|P|$. D'après la Proposition 4, on a $:|P \circ g|=|P|$. Ceci implique qu'il existe $a$ complexe de module un tel que : $P \circ g=a P$ et $a$ vaut 1 car $g$ est tangent à l'idendité.

On particularise maintenant la situation en prenant pour $P$ un produit de $n \geq 3$ formes linéaires $f_{i}$ sur $\mathbb{C}^{2}$ non proportionnelles deux à deux. Quitte à faire une transformation linéaire, on peut supposer $f_{1}=x$ et $f_{2}=y$ et les $f_{i}$ de la forme $x-a_{i} y$ pour $i \geq 3$. On fixe $s$ complexe non nul de module plus petit que 1 . On considère $g$ comme précédemment et on note $g_{1}$ et $g_{2}$ ses deux composantes. Pour $s$ complexe non nul de module plus petit que 1 on note $Z_{s}$ la courbe algébrique affine définie par $\{P=s\}$. La restriction de la fonction $h=g_{1} / g_{2}$ à cette courbe ne prend pas les valeurs $0, \infty, a_{3}, \ldots, a_{n}$. Ceci se déduit 
de l'égalité $P \circ g=P$. On notera aussi que pour $s$ assez petit en module, cette fonction n'est pas constante. Dans le cas contraire, on aurait toujours par l'égalité précédente $g_{1}$ et $g_{2}$ constant sur $Z_{s}$, ce qui est contradictoire avec $g$ tangent à l'idendité en l'origine. On supposera cette condition remplie dans la suite. La complétion projective $\bar{Z}_{s}$ de $Z_{s}$ est lisse et s'obtient à partir de $Z_{s}$ en lui ajoutant $n$ points à l'infini. D'après le grand théorème de Picard et en utilisant $n \geq 3$, la fonction $h$ se prolonge en une fonction holomorphe (encore notée $h$ ) à valeurs dans la droite projective $\mathbb{P}^{1}$. Chaque valeur $0, \infty, a_{3}, \ldots, a_{n}$ est alors prise par $h$ en un des $n$ point à l'infini et chacun de ces points correspond à une des directions $\left\{f_{i}=0\right\}$. En considérant les pôles et les zéros de $h$, on déduit qu'il existe deux entiers $p$ et $q$ positifs et premiers entre eux ainsi qu'une constante $d$ tels que : $h^{p}=d\left(f_{i} / f_{j}\right)^{q}$. A priori tous ces éléments dépendent de $s$. Mais on voit qu'ils en sont indépendants en remarquant que les possibilités sont dénombrables et donc pour un ensemble non dénombrable de $s$ on aura les mêmes choix. Un argument élémentaire d'analycité permet de conclure.

La relation $P \circ g=P$ jointe au fait que $g$ est tangente à l'idendité permet de montrer qu'il existe $n$ fonctions holomorphes $l_{i}$ définies sur $X$ et telles que $g_{1}=x l_{1}, g_{2}=y l_{2}, g_{3}=\left(x-a_{3} y\right) l_{3}$, etc. $\ldots$ où on a posé $g_{3}=g_{1}-a_{3} g_{2}$, etc. ... . Toujours d'après la relation $P \circ g=P$, on a que le produit des $l_{i}$ vaut 1 et en particulier les $l_{i}$ ne s'annulent pas. De ces remarques, il s'ensuit d'abord que $p$ et $q$ sont égaux à 1 , et donc que $a_{1}=a_{2}$. En raisonnant ensuite sur $g_{1}$ et $g_{3}$ etc. ..., on voit que tous les $a_{i}$ sont égaux, ce qui va impliquer qu'ils sont tous égaux à un. Ceci termine la preuve.

Remarque (suggérée par Daniel Naie). En utilisant le théorème de Riemann-Hurwitz, on peut montrer dès le départ que $p$ et $q$ sont égaux à un.

On a aussi la proposition suivante :

Proposition 7. Les seuls domaines disqués pseudo-convexes de $\mathbb{C}^{2}$ qui contiennent une courbe entière non constante différente d'une droite vectorielle sont les domaines exceptionnels du premier type.

Remarque. Ceci implique que les domaines disqués pseudo-convexes de $\mathbb{C}^{2}$ qui sont Brody-hyperbolique (i.e. qui ne contiennent pas des courbes entières non constantes) sont les domaines qui ne contiennent pas de droite vectorielle comme par exemple ceux de l'Exemple 2. Ceci se montre en utilisant la proposition et en remarquant que tous les domaines du premier type contiennent une droite vectorielle. Notons que 
des exemples de domaines disqués Brody-hyperboliques et non hyperboliques (c'est à dire non bornés) avaient été donnés [9]. Ces exemples n'étaient pas pseudo-convexes.

Preuve de la Proposition 7: Supposons l'existence dans un domaine disqué pseudo-convexe $X$ de $\mathbb{C}^{2}$ d'une courbe entière qui ne soit pas contenue dans une droite vectorielle. Le cas où cette courbe est situé dans $\left\{j_{X}=0\right\}$ a déjà été traité et alors $X=\mathbb{C}^{2}$. Sinon, cette courbe ne passant pas par l'origine, on associe à chacun de ses points la direction dans $\mathbb{P}^{1}$ de la droite joignant l'origine à ce point. Notons que ces directions ne sont pas bonnes car en chacun des points associé à une telle direction, il passe un disque analytique qui est la courbe entière ou un homothétique. En revenant à l'application entière correspondant à la courbe, on voit qu'on a une application holomorphe de $\mathbb{C}$ dans $\mathbb{P}^{1}$. Par le théorème de Picard, l'image évite au maximum deux points. Donc on a nécessairement un domaine exceptionnel du premier type.

Donnons pour terminer une autre proposition qui caractérise les domaines exceptionnels du premier type.

Proposition 8. Un domaine disqué pseudo-convexe $X$ dans $\mathbb{C}^{2}$ est exceptionnel du premier type si et seulement si il existe un automorphisme linéaire $A$ tel que $A(X)=X$ et $A$ n'est pas semblable à une matrice diagonale dont les valeurs propres sont de module un.

Preuve: On vérifie aisément qu'un domaine exceptionnel du premier type a des automorphismes linéaires vérifiant les conditions de la proposition. Pour voir que la condition est nécessaire, on regarde ce qui peut se passer pour $A$ (à conjugaison linéaire près). On note $a$ et $b$ les deux valeurs propres de $A$.

1) $|a|>1,|b|>1$ ou $|a|<1,|b|<1$.

Quitte à remplacer $A$ par son inverse, on peut supposer qu'on est dans le premier cas. Il est alors facile de voir que pour n'importe quelle boule ouverte $B$ centrée à l'origine, l'union des $A^{n}(B)$ (n variant dans $\mathbb{N}$ ) est tout $\mathbb{C}^{2}$. On a donc $X=\mathbb{C}^{2}$ dans ce cas.

2) $|a|<1 \leq|b|$.

On remarque que la fonction de Green d'un domaine invariant par $A$ est elle aussi invariante par $A$. Ceci implique que la mesure $m$ associée sur $\mathbb{P}^{1}$ est invariante par l'homographie $h: z \rightarrow c z$ associée à $A$ avec $|c|<1$ (ici la droite projective est vue comme $\mathbb{C} \cup \infty$ ). En prenant un disque $D$ de rayon suffisemment petit centré en un point différent de 0 et $\infty$, on voit que les $c^{n}(D)$ sont disjoints 
pour $n$ variant dans $\mathbb{Z}$. Comme la mesure $m$ est finie, ceci implique qu'elle ne peut que charger l'ensemble $\{0, \infty\}$. On est bien dans une situation exceptionnelle du premier type.

3) Il reste le cas où $a$ et $b$ sont égaux et de module 1, et la matrice $A$ triangulaire. On peut se ramener au cas $a=b=1$ et on raisonne comme pour 2). Ici la mesure $m$ est invariante par la translation $z \rightarrow z+1$. Le seul point qui peut être chargé est le point à l'infini. On est donc à équivalence linéaire près dans le cas de $\mathbb{C}^{2}$ ou dans celui de $\mathbb{C} \times \Delta$.

Remerciements. Je tiens à remercier F. Berteloot et J. P. Vigué pour l'intéret qu'ils ont porté à ce travail ainsi que pour les encouragements qu'ils m'ont prodigué. Je remercie également C. T. Dinh, M. Nicolau, K. Oeljeklaus et les collègues de l'Université de Rome pour les discussions que nous avons eues à ce sujet.

\section{Références}

[1] M. ABATE, "Iteration theory of holomorphic maps on taut manifolds", Research and Lecture Notes in Mathematics. Complex Analysis and Geometry, Mediterranean Press, Rende, 1989.

[2] Th. J. BARTh, The Kobayashi indicatrix at the center of a circular domain, Proc. Amer. Math. Soc. 88(3) (1983), 527-530.

[3] F. Berteloot et G. Patrizio, A Cartan theorem for proper holomorphic mappings of complete circular domains, Adv. Math. 153(2) (2000), 342-352.

[4] H. Cartan, Les fonctions de deux variables complexes et le problème de la représentation analytique, J. Math. Pures Appl., ge serie 10 (1931), 1-114.

[5] J. E. Fornæss et N. Sibony, Complex dynamics in higher dimensions, Notes partially written by Estela A. Gavosto, in: "Complex potential theory" (Montreal, PQ, 1993), NATO Adv. Sci. Inst. Ser. C Math. Phys. Sci. 439, Kluwer Acad. Publ., Dordrecht, 1994, pp. 131-186.

[6] R. C. Gunning, "Introduction to holomorphic functions of several variables. Vol. II. Local theory", The Wadsworth \& Brooks/Cole Mathematics Series, Wadsworth \& Brooks/Cole Advanced Books \& Software, Monterey, CA, 1990. 
[7] M. HeRvé, Quelques propriétés des applications holomorphes d'une boule à $m$ dimensions dans elle-même, in: Séminaire d'Analyse, dirigé par P. Lelong, 1962/63, Exp. 3, Secrétariat mathématique, Paris, 1963, $10 \mathrm{pp}$.

[8] M. KLIMEK, "Pluripotential theory", London Mathematical Society Monographs. New Series 6, Oxford Science Publications. The Clarendon Press, Oxford University Press, New York, 1991.

[9] S. Kobayashi, "Hyperbolic complex spaces", Grundlehren der Mathematischen Wissenschaften 318, Springer-Verlag, Berlin, 1998.

[10] A. Sadullaev, Schwarz's lemma for circular domains and its application, (Russian), Mat. Zametki 27(2) (1980), 245-253, 318.

[11] M. SuzukI, The intrinsic metrics on the circular domains in $\mathbb{C}^{n}$, Pacific J. Math. 112(1) (1984), 249-256.

[12] E. Casadio Tarabusi et S. Trapani, Envelopes of holomorphy of domains in $\mathbb{C}^{n}$, in: "Complex analysis" (Wuppertal, 1991), Aspects Math. E17, Vieweg, Braunschweig, 1991, pp. 70-76.

[13] W. Rudin, The fixed-point sets of some holomorphic maps, Bull. Malaysian Math. Soc. (2) 1 (1978), 25-28.

[14] E. Vesentini, Complex geodesics, Compositio Math. 44(1-3) (1981), 375-394.

Département et Laboratoire de Mathématiques

Université d'Angers

2 Boulevard Lavoisier

49045 Angers cedex 01

France

E-mail address: Jean-Jacques.Loeb@univ-angers.fr

Primera versió rebuda el 6 d'abril de 2005, darrera versió rebuda el 2 de novembre de 2005 . 